\title{
Research Paper: Evaluation of the Effects of Two Types of Foot Rockers on the Temporal- Spatial Gait Parameters in Diabetic Patients
}

\author{
*Zeinab Rezaeian ${ }^{1}$, Mohammad Taghi Karimi ${ }^{1}$, Arezoo Eshraghi ${ }^{2}$, Niloofar Fereshtenejad ${ }^{1}$
}

1. Department of Orthotics and Prosthetics, Musculoskeletal Research Center, Isfahan University of Medical Sciences, Isfahan, Iran. 2. Department of Biomedical Engineering, Faculty of Engineering, University of Malaya, Kuala Lumpur, Malaysia.

Cttation: Rezaeian Z, Karimi MT, Eshragh A. [Evaluation of the Effects of Two Types of Foot Rockers on the Temporal-Spatial Gait Parameters in Diabetic Patients (Persian)]. Journal of Rehabilitation. 2016; 17(2):168-177. http://dx.crossref.org/ 10.21859/jrehab-1702168

: http://dx.crossref.org/ 10.21859/jrehab-1702168

Received: 12 Feb. 2016 Accepted: 15 May 2016

Keywords:

Diabetic neuropa-

thy, Toe rocker, Heel rocker, Temporalspatial parameters of gait, Force-time integral

\section{ABSTRACT}

Objective Foot ulcer is one of the main challenges of diabetic patients influencing their abilities to stand and walk. Various methods have been suggested to decrease the loads applied to the foot in this group of patients; most methods were not deemed successful and could only be used temporarily. Rocker shoes are recommended for foot ulcer treatment. Based on the available literature, it is still controversial whether or not the forces applied to the foot differ between diabetic and normal subjects. Moreover, it is not well understood which kind of rocker (heel or toe rocker) is more successful in decreasing the applied forces to the foot. Therefore, this study aimed to compare the loads applied on the foot in diabetic subjects and normal subjects and to determine the influence of rockers on the load of foot. The main hypothesis associated with this study was that heel and toe rockers had similar effects on the force applied on the foot in diabetic subjects.

Materials \& Methods In this interventional, and quasi-experimental study, 20 healthy and 20 diabetic subjects were recruited. The sample size was determined based on the average number of the subjects in the previous similar studies. The diabetic subjects were selected from the patients referred to foot clinics in Rehabilitation School of Isfahan University of Medical Sciences. A qualysis motion analysis system with a Kistler force plate $(50 \times 60 \mathrm{~cm})$ was used to record the temporal-spatial gait parameters and forces applied on the leg during walking. The subjects were asked to walk with a comfortable speed along a level surface in a gait lab. The parameters such as temporal-spatial gait parameters, peaks of ground reaction forces, and force-time integral were used for analysis. The difference between the mean values of the parameters and between the subjects' effects were evaluated by use analysis of variance with repeated measures test. The statistical analysis was done by SPSS17 and with significant level of 0.05.

Results There was a significant difference between normal and diabetic foot subjects regarding cadence, stride length, and percentage of stance phase $(P<0.05)$. Although the walking speed of diabetic subjects was less than that of normal subjects, the difference was not significant $(P>0.06)$. There was a significant difference between the peak of the forces (anteroposterior [fy1, fy2], medio lateral [fx], and vertical [fz1, $\mathrm{fz} 2, \mathrm{fz} 3]$ ) applied on the leg in normal and diabetic foot. The type of rocker did not affect the spatiotemporal gait parameters $(P<0.05)$, while exhibited significant effect on the peak anteroposterior forces applied to the leg $(P<0.04)$. The mean value of the force-time integral of vertical component of ground reaction force varied based on the side and group $(P<0.04)$.

Conclusion The results of this study showed that the force-time integral of vertical component of the ground reaction force increased significantly in subjects with diabetes, which is the main cause of foot ulcers. Although, heel and toe rockers did not influence the force applied to the foot or the force-time integral, it is recommended to use rocker shoes with wide base of support to increase the dynamic stability while decreasing the foot pressure. The main limitation is that the immediate effect of use of rocker was studies in this study. It is recommended to check the effect of rockers interventions after a certain period of their use.

\section{* Corresponding Author:}

Zeinab Rezaeian, MSc.

Address: Department of Orthotics and Prosthetics, Musculoskeletal Research Center, Isfahan University of Medical Sciences, Isfahan, Iran.

Tel: +98 (31) 42649366

E-Mail: rezaeian.zeinab.236@gmail.com 


\title{
بررسى تأثير دو نوع راكر كفش بر متغيرهاى زمانى -مكانى راهرثتن در بيماران ديابتى
}

\author{
"زينب رضاييان'، محمدتقى كريمى'، آرزو اشراقى'، نيلوفر فرشتهنراد' \\ ا - كروه ارتويدى فنى، دانشكده توانبخشى، مركز تحقيقات اختلالات اسكلتى و عضلانى، دانشُكاه علوم يزُشكى اصفهان، اصفهان، ايران.

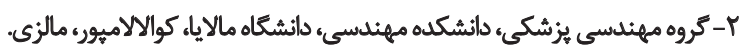

\begin{abstract}
Q

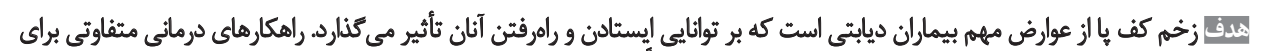

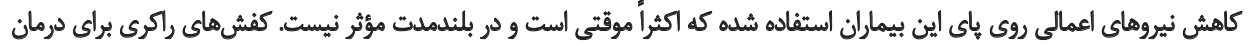

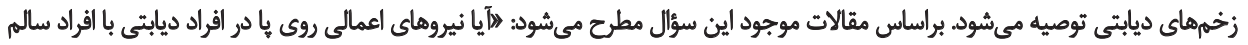

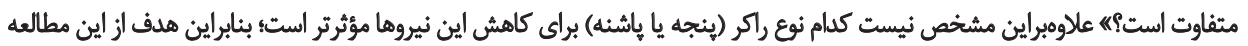

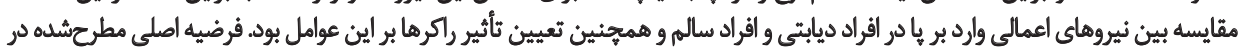

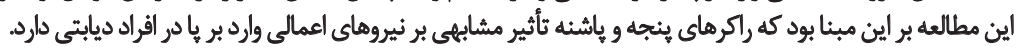

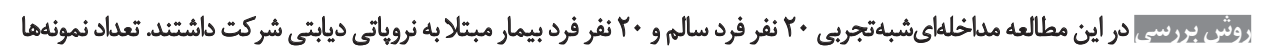

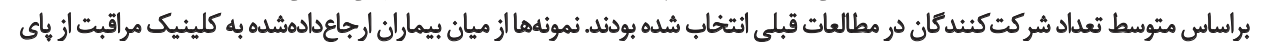

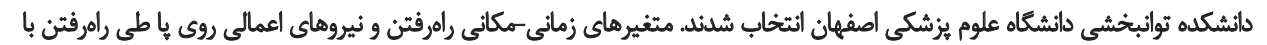

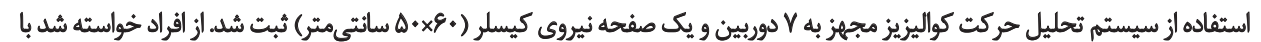

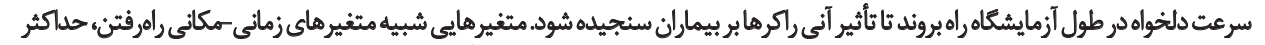

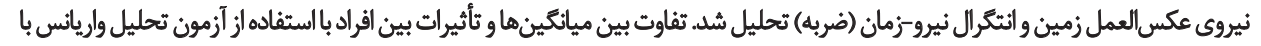

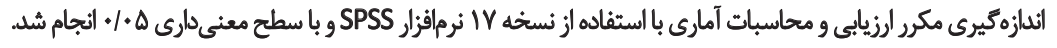

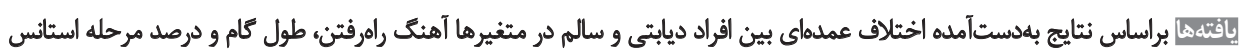

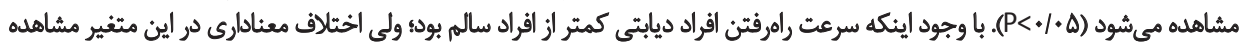

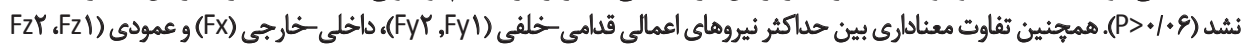

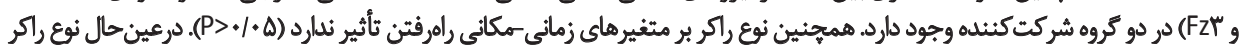

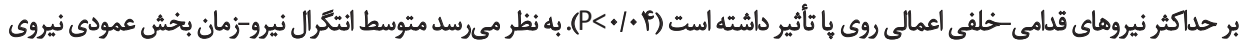

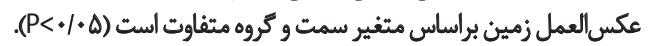

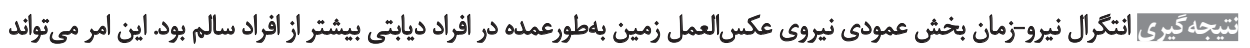

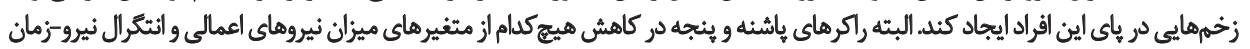

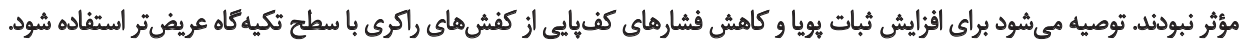

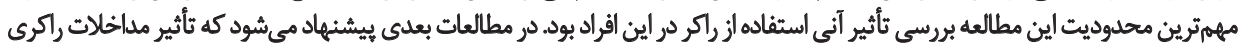
بعد از يك دوره استفاده ارزيابى شوده.
\end{abstract}

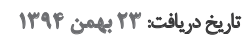

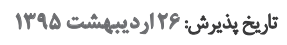

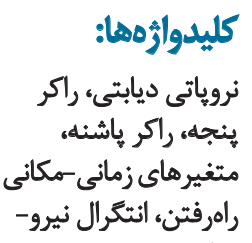

زمان

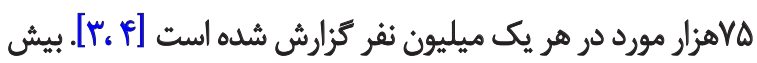

مقلدم

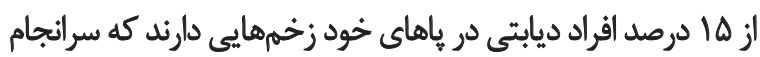

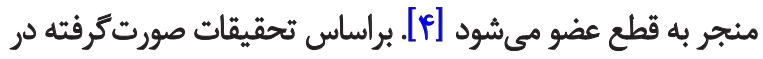

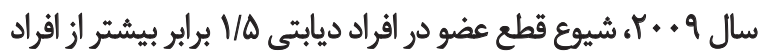

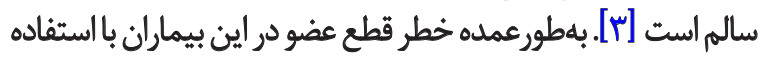

ديابت و زخمهاي ناشى از آن، يكى از مهمترين بيمارىهايیى است

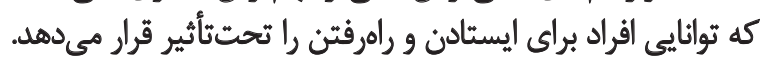

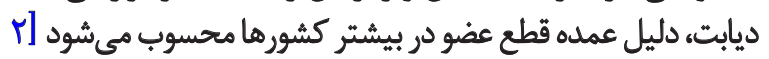

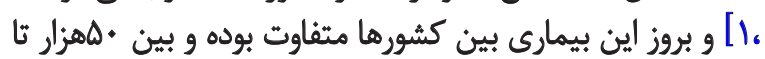

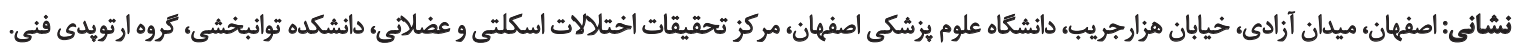

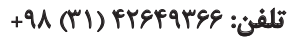
رايانامه: rezaeian.zeinab.236@gmail.com 


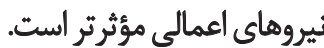

هدف از اين مطالعه مقايسه بين نيروهاي اعمالي وارد بر يا دادر افراد

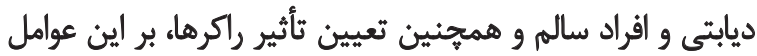

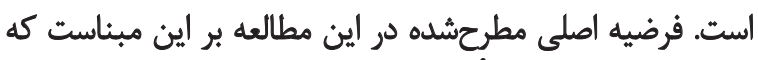

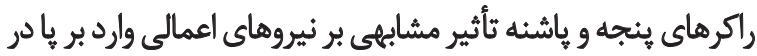

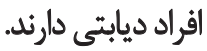

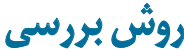

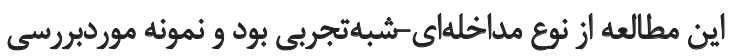

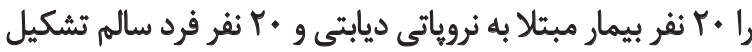

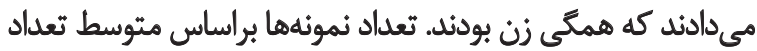

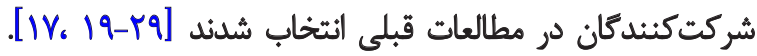

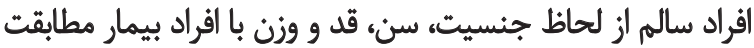

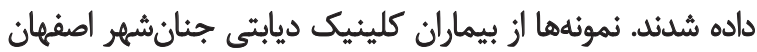

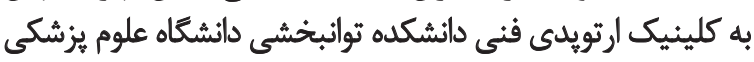

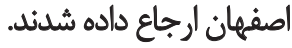

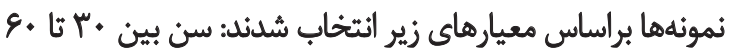

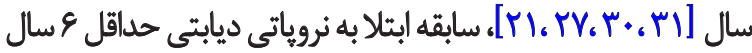

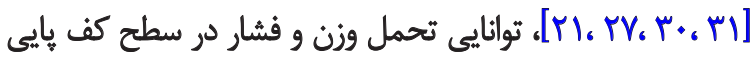

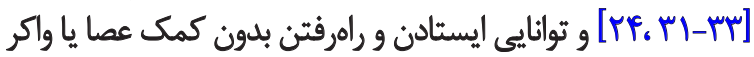

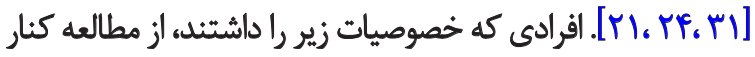

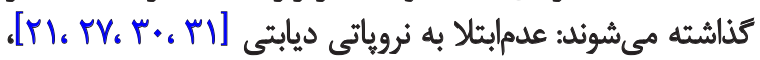

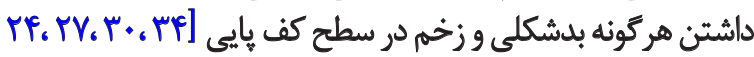

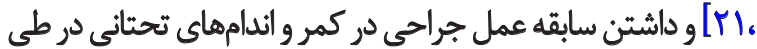

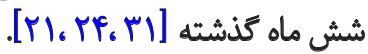

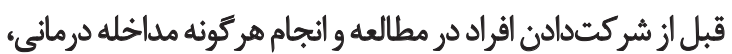

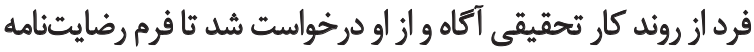

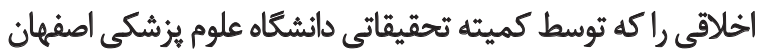

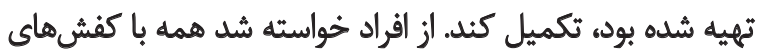

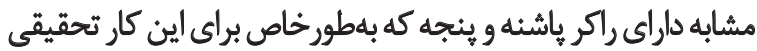
ساخته شده بود، راه بروند.

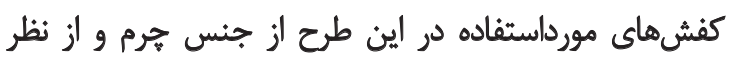

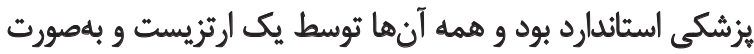

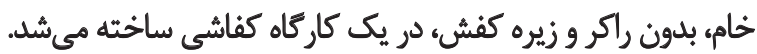

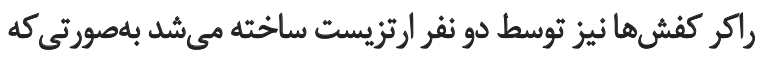

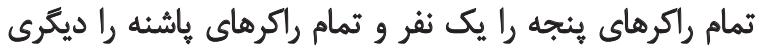

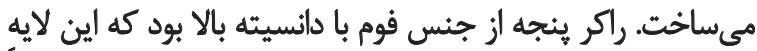

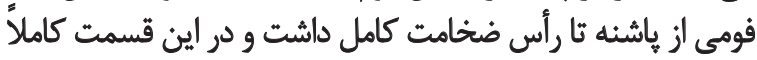

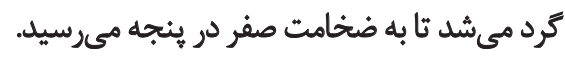

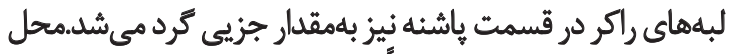

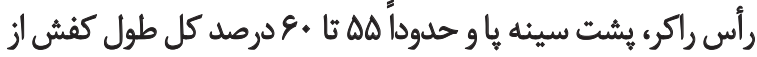

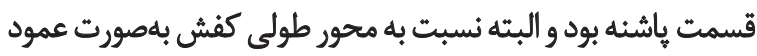

$$
\text { از راهكارهاى درمانى مناسب كاهش هي يابد. }
$$

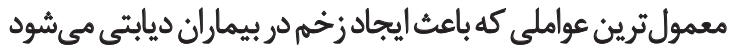

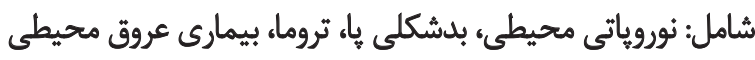

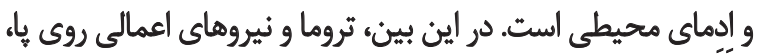

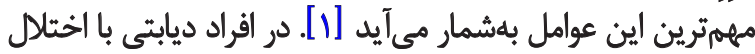

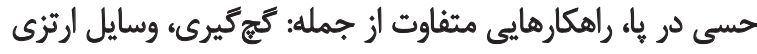

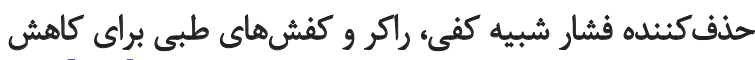

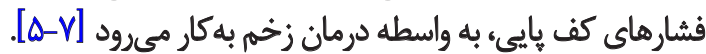

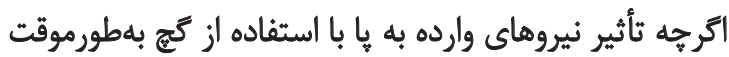

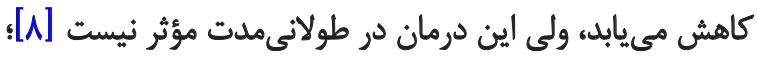

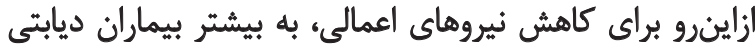

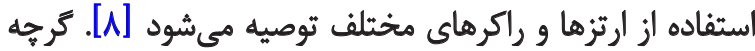

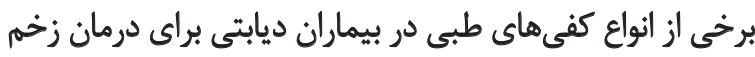

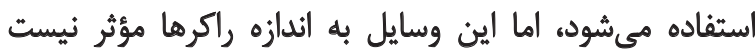

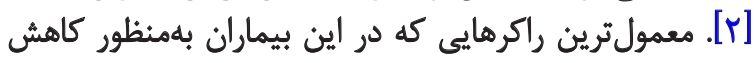

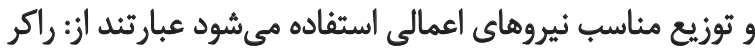

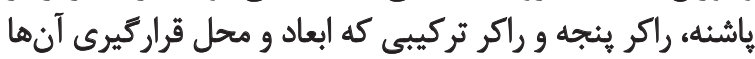

براساس نياز بيمار تغيير مى كند [9].

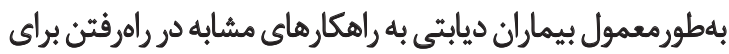

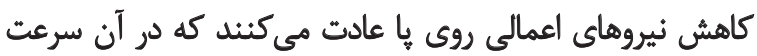

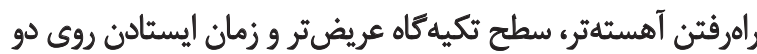

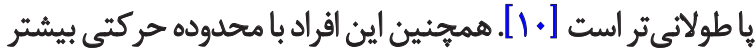

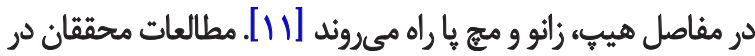

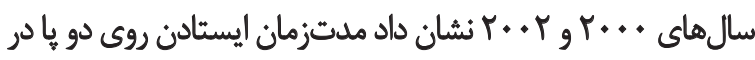

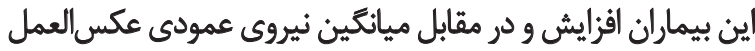

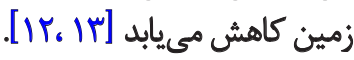

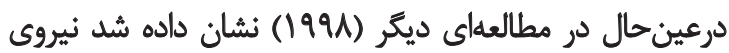

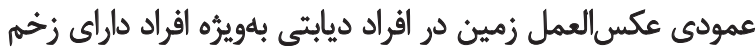

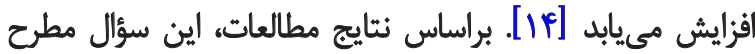

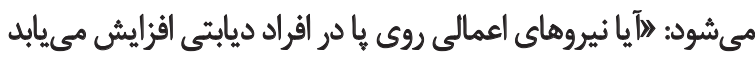

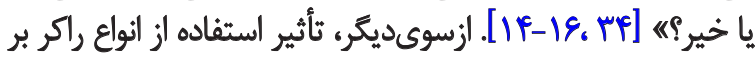

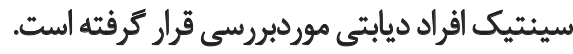

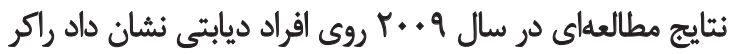

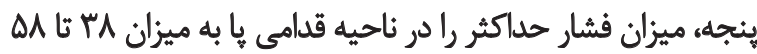

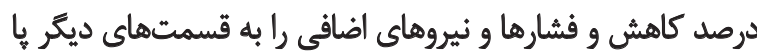

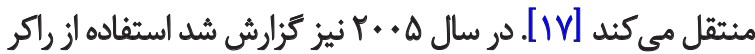

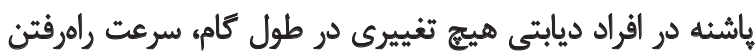

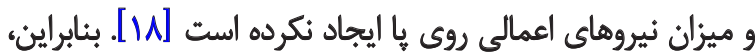

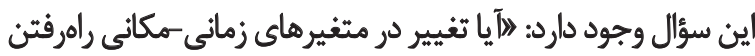

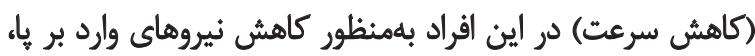

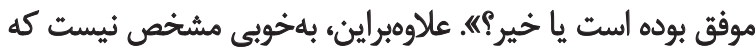

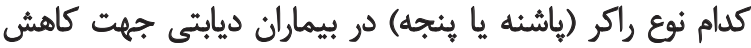




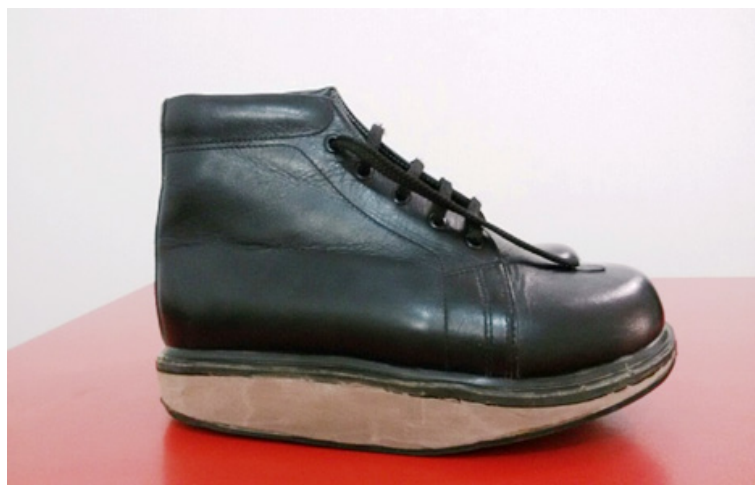

ب) (اكر ينجه

توانبخننى

قبل از شروع آزمون با انجام قرعهشيى، نوع كفش راكرى كه فرد

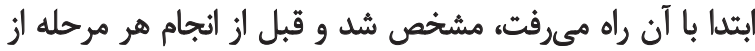

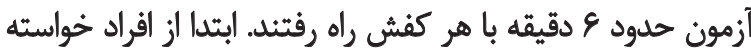

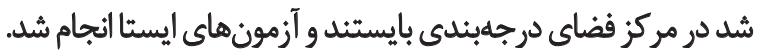

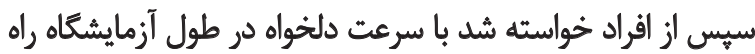

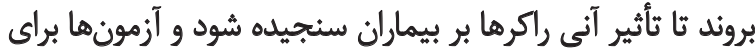

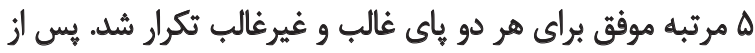

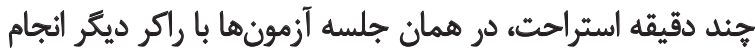

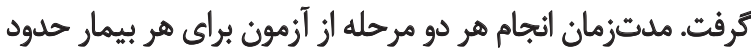

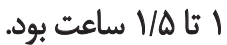

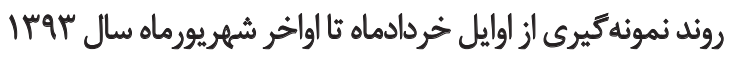

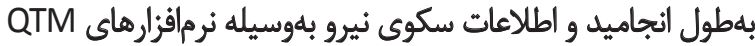

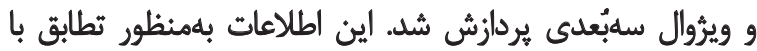

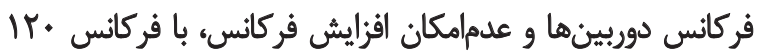

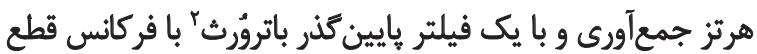

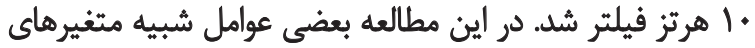

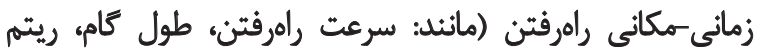

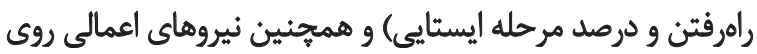

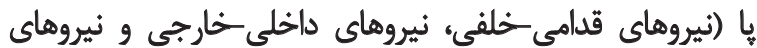

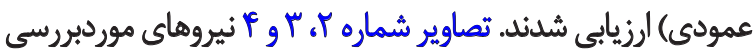

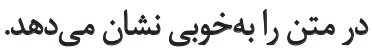

براى مقايسه مشخصات فردى بين دو كروه شركت منثندها.

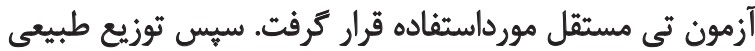

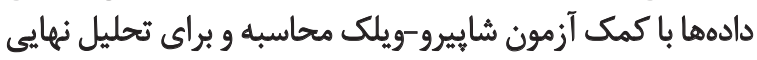

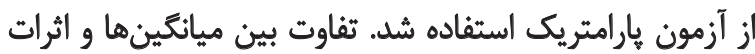

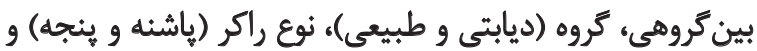

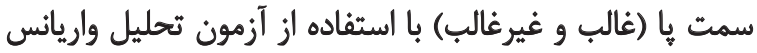

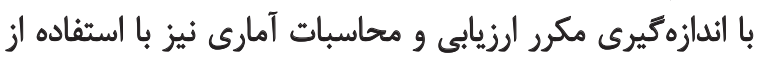

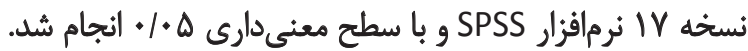

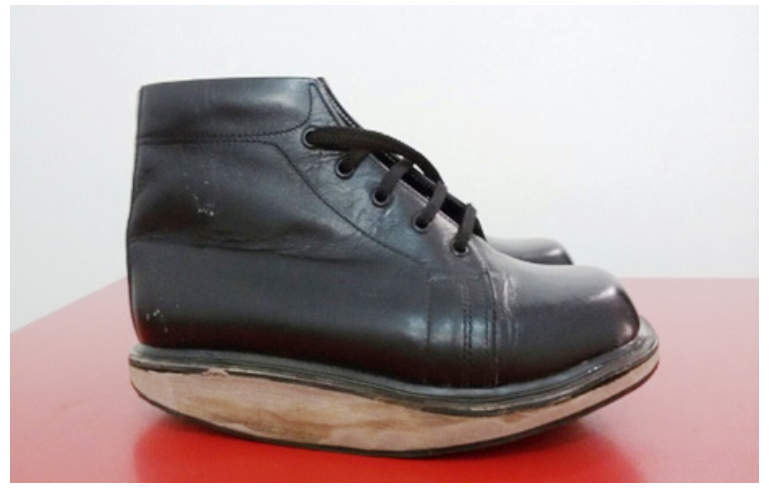

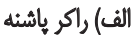

تصوير ا. كفشهاى راكرى مورداستفاده در اين مطالعه.

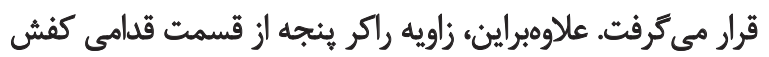

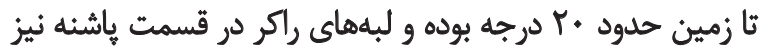

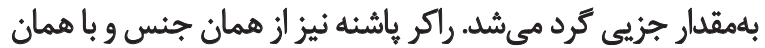

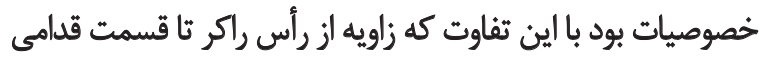

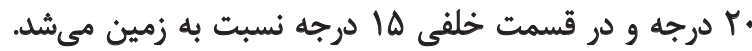

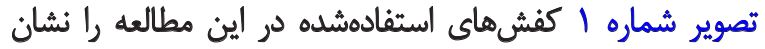

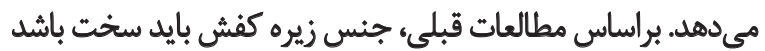

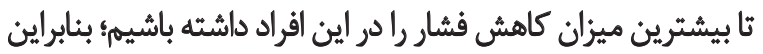

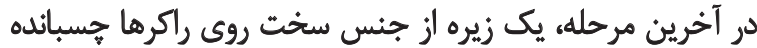

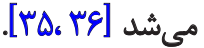

يك سيستم تحليل حركت كواليزيز (سرى يك ساحت كشور

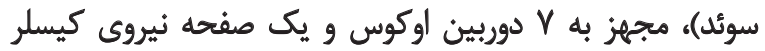

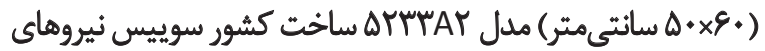

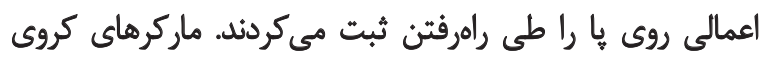
قايل If

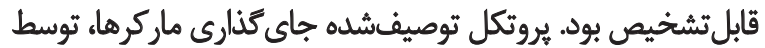
كروه مهندسى دانشَّاه استراتكلايد' ابداع شده است.

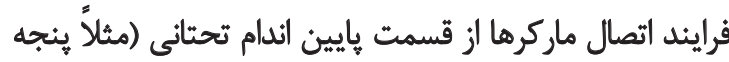

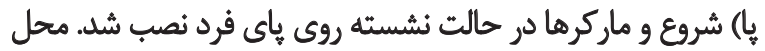

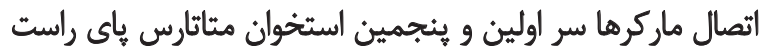

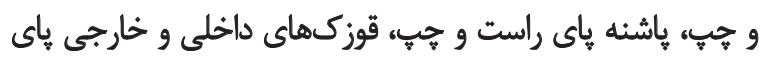

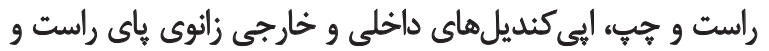

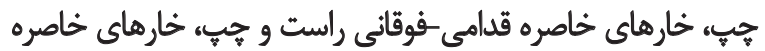

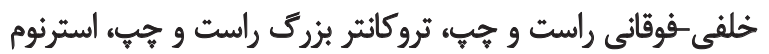

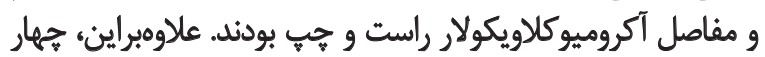

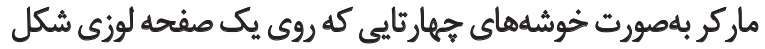

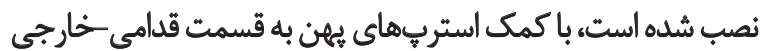
ساق و ران ياها متصل شدند [rV] در ابتداى جلسه، افراد براى انجام آزمون راهرفتن آموزش ديدند. 


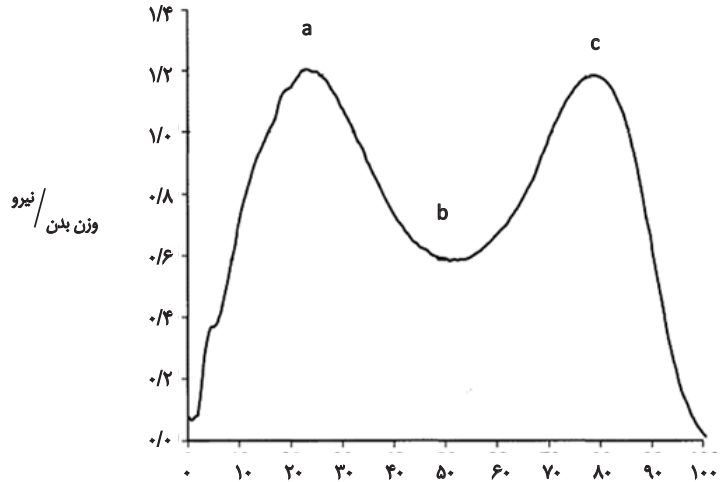

ورهد مرجله ايستياين

توانبخننى

تصوير T. نيرو در جهت عمودى در طى راهرفتن معمولى.

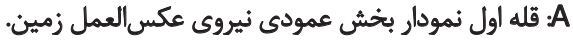

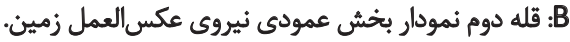
C قله سوم نمودار بخش عمودى نيخ عمودى نيروى عكس العمل زمين.

تأثير عوامل كروه (ديابتى -طبيعى)، راكر (ياشنه-ينجه) و سمت

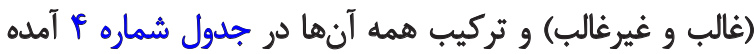

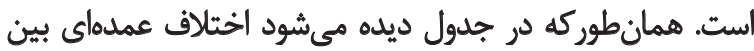

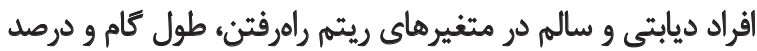

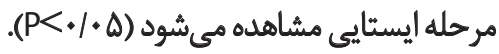

با وجود اينكه سرعت راهرفتن افراد ديابتى كمتر از افراد سالم بود إداد

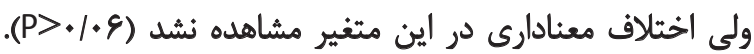

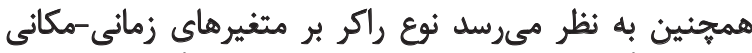

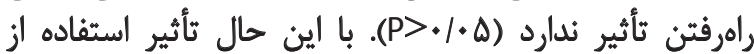

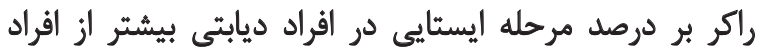

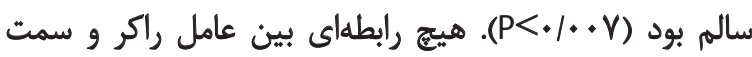

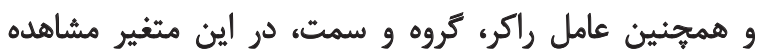

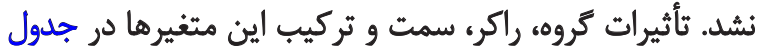

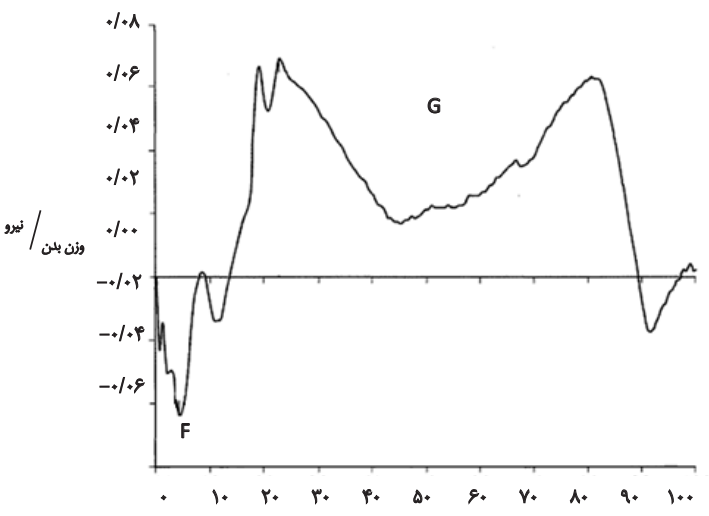

$$
\text { درصد هرحله إيستاييى }
$$

توانبخنتى

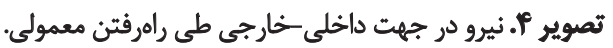

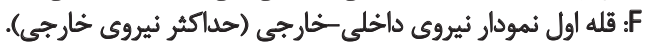

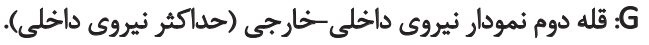

يافتهنا

مشخصات فردى افراد شركت كننده در تحقيق، در جدول شماره

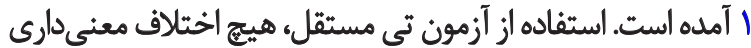

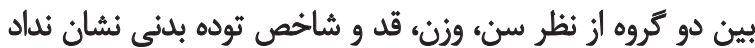

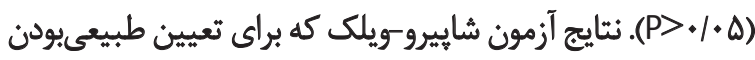

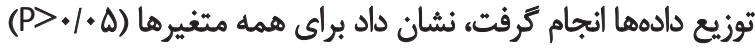

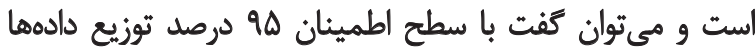

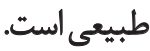

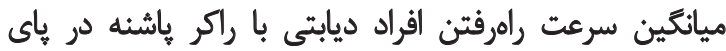

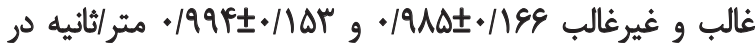

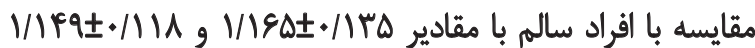

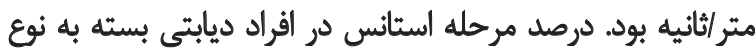

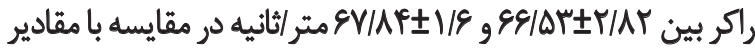

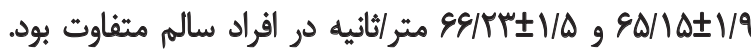

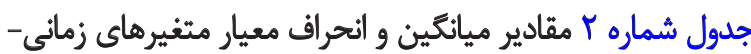

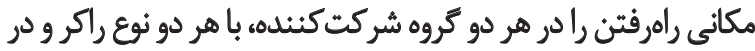
هر دو سمت نشان مي دهد.

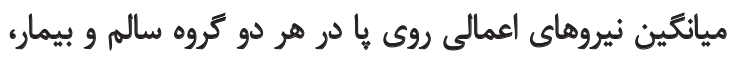

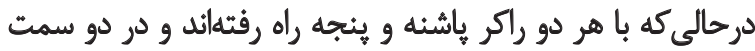

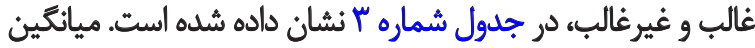

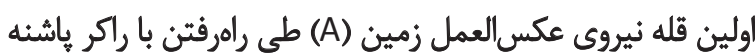

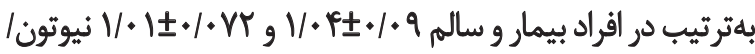

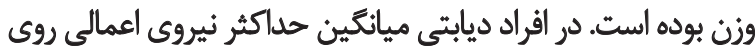

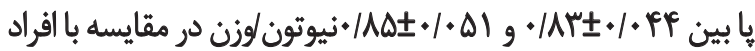

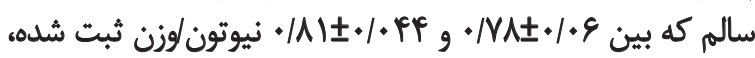
متثفاوت أستث.

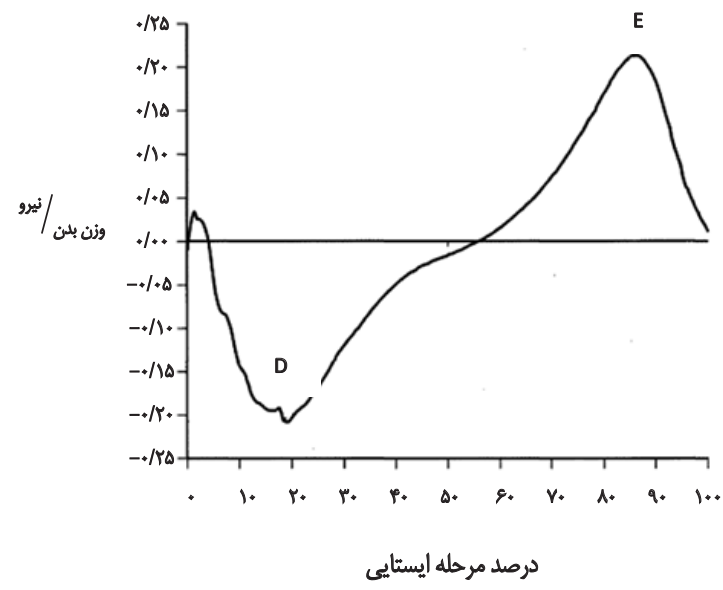

توانبخنتى

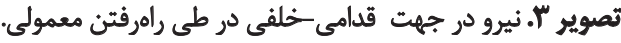

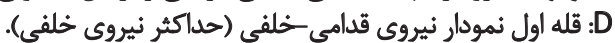

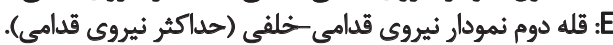


جدول ا. مشخصات فردى افراد شركتكنئه.

\begin{tabular}{|c|c|c|c|}
\hline P-value & كروه افراد طييى & كروه بيماران & مثغير موردبررسى \\
\hline . $/ A T$ & $P \& / \Delta \pm / \Delta / 9 q$ & $P V / F \pm E N E 9$ & سن (سال) \\
\hline.$/ 4$ & $V+N \pm E N / \cdot 1$ & $n \in / / r \pm v e / q \Delta$ & وزن (كيلوكرم) \\
\hline$\cdot / A Y$ & $V / \pm \Delta F / \cdot r$ & $1 /= \pm \Delta \% /+\varphi$ & قد (متر) \\
\hline.$/ 4$ & YNTEqA/AY & $r+/ \Delta \pm \Delta \varphi / C$ & شاخص توده بدنى (كيلوكر / متر)') \\
\hline
\end{tabular}

توانبخننى

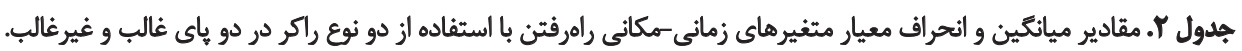

\begin{tabular}{|c|c|c|c|c|c|c|}
\hline سرعت (مثر/ثانيه) & درصد مرحله أيستايي (ب سيكل & طول كام (مثر) & ريتم راهرقئن (قدم/ & سمت & راكر & كروه \\
\hline$+1+ \pm 2 \Delta / 198$ & $\mathscr{S} / T \pm \Delta T / A T$ & $1 / \pm 1 f+1 \cdot u$ & $1+r / 1 \cdot \pm 19 / v g$ & غالب & \multirow[b]{2}{*}{ بالشنه } & \multirow{4}{*}{ ديابتى } \\
\hline 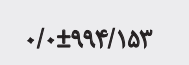 & $E \notin / Y \pm A V / T H \Delta$ & $1 / . \pm .91 / T F Y$ & $1 \cdot r / 1 \cdot \pm 9 \Delta / M^{6}$ & غيرغالب & & \\
\hline$\% \pm q 4 \sqrt{ }$ & $\mathscr{E} / \backslash \pm \wedge F / 8$ & $V \cdot \pm|r| r \mid \cdot e$ & 1.1/11Eeg/1A & غالب & \multirow{2}{*}{ يُنجه } & \\
\hline $1 /+ \pm \cdot T V / 190$ & $S V / \triangle \pm M Y / 8$ & $1 / \pm \pm 1 \gamma+1 \cdot \lambda r$ & $1 \cdot F / I \pm N F / T A$ & غيرغالب & & \\
\hline $1 /+ \pm \mid F \Delta / I T \Delta$ & $g F / \Lambda \pm T r / \Delta$ & $1 / . \pm T r r / \cdot F \Delta$ & $\| r / q \pm V N / F$ & غالب & \multirow{2}{*}{ با باشته } & \multirow{4}{*}{ طبيعى } \\
\hline $1 / . \pm 1 F 9 / 111$ & $8 \Delta / \cdot \pm \& V / q$ & V.Etratres & $\| Y / A \pm \lambda F / q$ & تغيرغالب & & \\
\hline$V / \pm|A T /| Y \mid$ & $8 \Delta / \backslash \pm \Delta T / M$ & $V \cdot \pm \pi r+1 \cdot \Delta \cdot$ & $\| F / M \cdot \pm \Delta V / V E$ & غالب & \multirow{2}{*}{ يُنجيه } & \\
\hline $1 / * \pm|9| / r+1$ & $q \Delta / \backslash \pm \backslash \Delta / q$ & $1 / \pm \pi m+.9 \%$ & $111 / M \pm+V / T$ & غيرغالب & & \\
\hline
\end{tabular}

توانبخننى

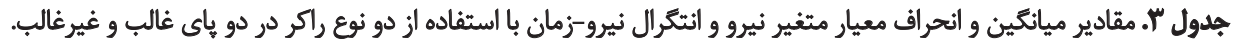

\begin{tabular}{|c|c|c|c|c|c|c|c|c|}
\hline 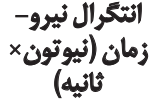 & F) F (وذن/نيوتون) & E) E (وزن/نيوتون) & D(وزن/نيوتون) & C (وزن/ثيوتون) & B) B (وزن/نيوتون) & A(وزن /نيوتون) & سمت & راكر \\
\hline.$/ . \pm \cdot \cdot 19 / . .19$ & $\cdot 1 \cdot \pm \cdot \Delta r / \cdot 9$ & $\circ|+ \pm| \& / . r \mid$ & . & $V \cdot \pm \cdot * / \cdot V I$ & 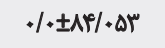 & $1 /+4 \pm .1 .9$. & غَالب & \multirow{2}{*}{ هإنشئ } \\
\hline$. \% \pm \cdot \cdot 14 \% \cdot .19$ & $* / * \pm+\mu r / *+1$ &. $\pm \pm 191 \cdot \pi$ & $\circ \pm \pm N \cdot \Delta$ & $V / * \pm \cdot r / \cdot F \Delta$ &.$/ \pm \lambda F / \cdot \Delta f$ & $|/ \cdot \pm+a| \cdot V$ & غيرغالب & \\
\hline$+/ + \pm * \cdot \Lambda_{\Delta} / *+Y_{*}$ & $+1+ \pm+\varphi N++9$ & $+/ \pm|Q|+Y q$ & $+/ \pm \pm|A|+M$ & $1 / \pm+.4 / .88$ & $+\mid+ \pm \wedge \Delta / \cdot \Delta 1$ & $V / \pm \pm+\Delta / \cdot V$ & غالب & \multirow{2}{*}{ هينجة } \\
\hline$• / \pm \bullet \cdot A Y / . \bullet r$. & $.10 \pm \cdot r \cdot 1 \cdot .9$ & $\cdot|+ \pm| Q \mid \cdot M$ & $.1 . \pm 1 \% 1 . \mu T$ & $1 / \pm .4 / .89$ & . $1 . \pm A M / \cdot \mu$ & $V \cdot \pm \cdot T / \cdot A r$ & غُيرغالب & \\
\hline.$/ . \pm * . v Q / . .14$ & $* 1+ \pm * \Delta Q / * 1$. & $* 1 * \pm 1 N \cdot 19$ & $\circ \pm \pm T f / . M f$ & $V \cdot \pm \cdot N \cdot F$ & $\% \pm Y N \cdot 8$. & $1 / \pm \pm \cdot 1 / \cdot n$ & تغالب & \multirow{2}{*}{ بإشئه } \\
\hline$* 1+ \pm \cdot \cdot V N \cdot \cdot 11$ & $\cdot 1+ \pm+* N \cdot \cdot V$ & $\triangle I \pm I V /+Y A$ & $* 1+ \pm 19 \cdot \pi$ & $\mid / \cdot \pm \cdot \Delta / \cdot \Delta \Delta$ & $+1 * \pm A+\mid \cdot \Delta 1$ & $V / E \pm \cdot Y / \cdot \Delta A$ & غيرغالب & \\
\hline$* / + \pm * v \varepsilon / \cdots q$ & $+1+ \pm+\Delta H /+. q$ & $+1+ \pm 1 \% /+18$ & $+1+ \pm \mid V /+4 E$ & $V / \pm \pm \cdot r / \cdot v F$ & $+1 * \pm A+1 * A F$ & $V / \pm+r / .9 V$ & غالب & \multirow{2}{*}{ ينجه } \\
\hline$. / \cdot \pm \cdot \cdot V N \cdot \cdot \| r$ & $\cdot 1 \cdot \pm \cdot \pi T / \circ+A$ &.$/ . \pm 1 \& / . Y \Delta$ & $\cdot 1 \cdot \pm W \cdot W$ & $1 / \cdot \pm \cdot \mathbb{\Delta} / \cdot \cdot \bullet$ & $\cdot 1 \cdot \pm A 1 / * A$ & $1 / \cdot \pm \cdot 1 / .80$ & غيرغالب & \\
\hline
\end{tabular}

توانبخننى

طى راهرفتن با راكر ئجه در هر دو كروه شركتكنئده كاهش يافته است.

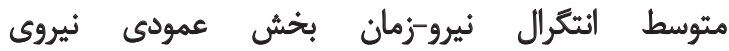

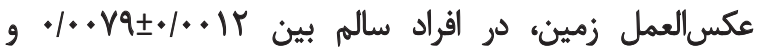

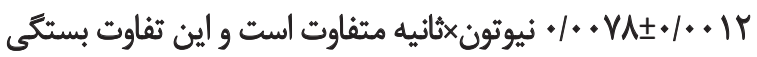

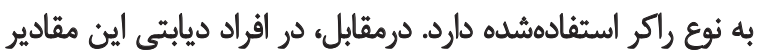

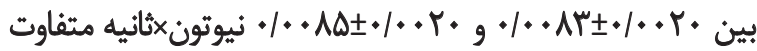

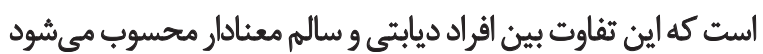

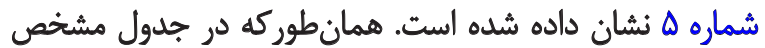

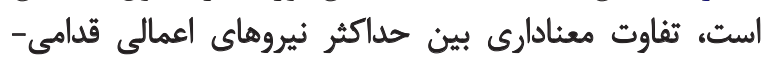

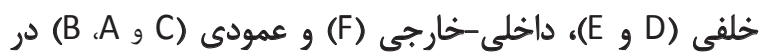

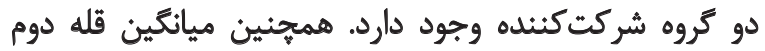

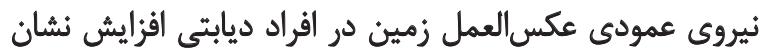

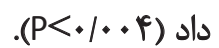

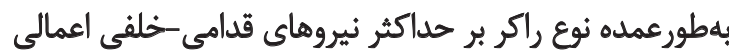

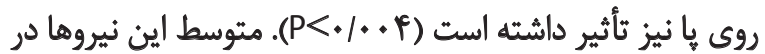


جدول fo. مقادير P-value متغيرهاي زمائى -مكانى راهرفتن با استفاده از دو نوع راكر در دو يايى غالب و غيرغالب.

\begin{tabular}{|c|c|c|c|c|}
\hline سرعت & درصد مرحله ايستاييى & طول كَام & ريتهم راهرفتن & عامل موردنظظر \\
\hline Sig. & Sig. & Sig. & Sig. & \\
\hline .1 .99 & - & $.1 .+r$ & $* / * \bullet$ & كروه - ل موه \\
\hline . ReT & - fer & . /eqr & - Mar & וرا \\
\hline.$/ r n$ & . MAT & $\cdot|A| \Delta$ & . $/ \Delta F A$ & سمت \\
\hline.$/ m$ & $\%+Y$ & $.108+$ & $+/ 99$ & كروهاتراكر \\
\hline 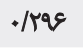 &.$/ p e r$ &.$/ T \Delta 1$ & $.1 . \pi$ & كرومانست \\
\hline.$/ T r A$ &.$/ 4 e r$ &.$/ 9 T V$ &.$/ M A$ & راكر \\
\hline ./TrI &.$/ \pi \Lambda$ &.$/ r q$ & .1 .14 & كروب* \\
\hline
\end{tabular}

جدول ه. مقادير P-value متغير نيرو و انتكرال نيرو-زمان با استفاده از دو نوع راكر در دو ياى غالب و غيرغالب.

\begin{tabular}{|c|c|c|c|c|c|c|c|}
\hline انتكرال نيرو-زمان & $\mathbf{F}$ & E & D & c & B & A & عامل موردنظر \\
\hline Sig. & Sig. & Sig. & Sig. & Sig. & Sig. & Sig. & \\
\hline $.1 .0 \%$ &.$/ . M F$ & $\cdots 1$ & .1110 & $\cdot m \cdot t$ & $.1 . .4$ & $\cdot N \cdot r$ & كروه \\
\hline 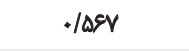 & - & .1 .01 & .1 .4$. & $.1 M$ &.$/ M r$ & $.19 .$. & راكر \\
\hline . $/$ DFA & + &.$/ \Delta r V$ & . & . & .1991 & IMq & سمت \\
\hline.$/ \pi 7$ &.$/ 994$ & $\cdot M$ & $\cdot r+9$ & $.1 . \pi$ & . $/ \Delta P Q$ & . MA & كوروها:راكر \\
\hline $.1 .+4$ & . Are & $.10 \cdot 0$ & . laVE & . THAQ &.$/ 119$ & $\cdot 18 \cdot \gamma$ & كروهاسمت \\
\hline$+/ D M$ & $+/ Y \Delta q$ &.$/ 9 \pi T$ &.$/ Y v \Delta$ & \% &.$/ \Delta 11$ & 1.98 & راكر \\
\hline .1 .1$. & . $/ \Delta V \Delta$ &.$/ 905$ & $\cdot M \cdot r$ & . & $\cdot / A \cdot Y$ & . TAS & 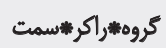 \\
\hline
\end{tabular}

افزايش در انتكرال نيرو-زمان در افراد ديابتى منجر به افزايش

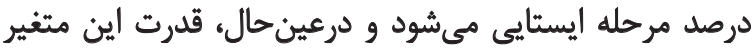

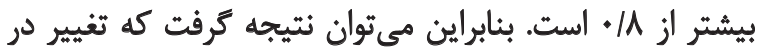

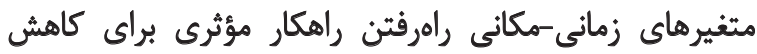

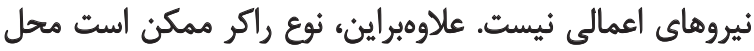

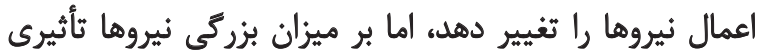

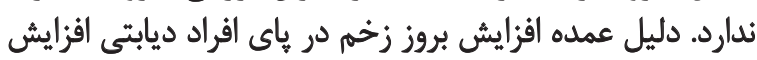

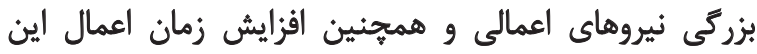
نيروها مي براشد [If

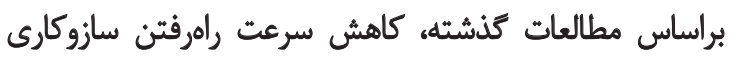

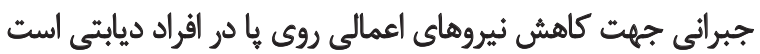

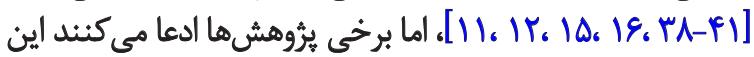

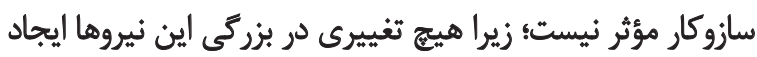

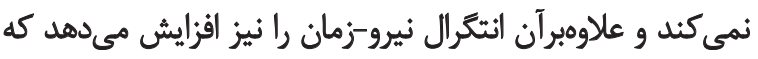

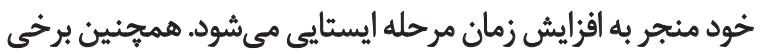

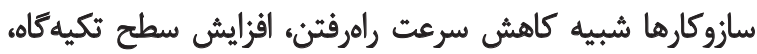

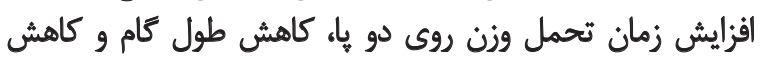

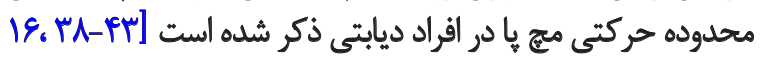

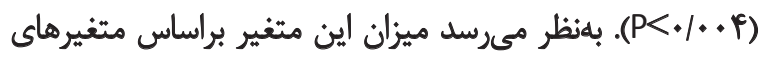

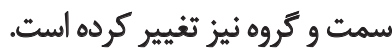

بحث

نتايج اين مطالعه نشان داد بهطورعمده در افراد ديابتى بيشتر

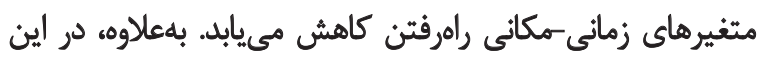

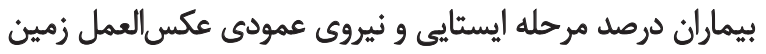

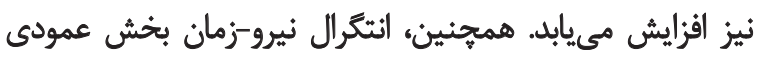

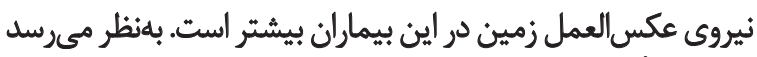

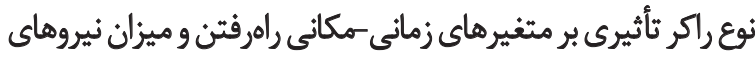

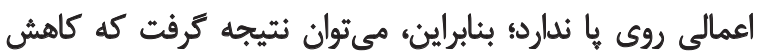

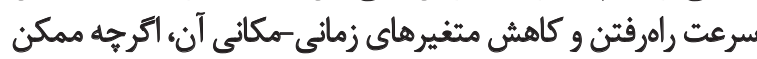

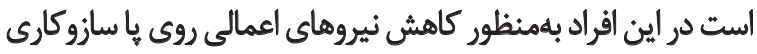

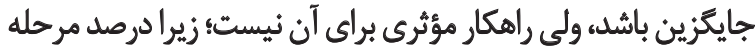

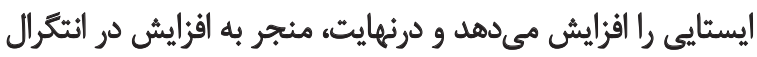

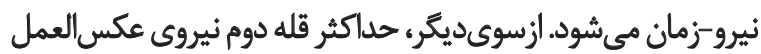

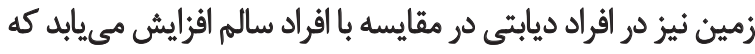

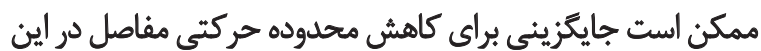




\section{References}

[1] Boulton A. The diabetic foot: grand overview, epidemiology, and pathogenesis. Diabetes \& Metabolism Research Review. 2008; 24(1):3-6.

[2] Paton J, Bruce G, Jones R, Stenhouse E. Effectiveness of insoles used for the prevention of ulceration in the neuropathic diabetic foot: a systematic review. Journal of Diabetes and its Complications. 2011; 25(1):52-62.

[3] Papanas N, Maltezos E. The Diabetic foot: a global threat and a huge challenge for Greece. Hippokratia. 2009; 13(4):199-204.

[4] Boulton AJM, Kirsner RS, Vileikyte L. Neuropathic diabetic foot ulcers. New England Journal of Medicine. 2004; 351(1):48-55.

[5] Kwon OY, Mueller MJ. Walking patterns used to reduce forefoot plantar pressures in people with diabetic neuropathies. Physical Therapy. 2001; 81(2):828-35.

[6] Hoar A. A case study for off-loading. Wound Care Canada. 2008; 6(1):58-59.

[7] Cavanagh PR. Therapeutic footwear for people with diabetes. Diabetes \& Metabolism Research \& Reviews. 2004; 20(1):51-55.

[8] Edmonds ME. 10-the diabetic foot: pathophysiology and treatment. Clinics in Endocrinology and Metabolism. 1986; 15(4):889-916.

[9] Saraswathy G, Gopalakrishna G, Das BN, Viswanathan V. Recent advances in diabetic foot care. In: Sebastian M, editor. Diabetes Mellitus and Human Health Care: A Holistic Approach to Diagnosis and Treatment. Oakville: Apple Academic Press; 2014, p. 315-56.

[10] Hutchins S, Bowker P, Geary N, Richards J. The biomechanics and clinical efficacy of footwear adapted with rocker profiles: evidence in the literature. Foot. 2009; 19(3):165-70.

[11] Petrofsky J, Lee S, Bweir S. Gait characteristics in people with type 2 diabetes mellitus. European Journal of Applied Physiology. 2005; 93(5):640-47.

[12] Sacco I, Amadio A. A study of biomechanical parameters in gait analysis and sensitive cronaxie of diabetic neuropathic patients. Clinical Biomechanics. 2000; 15(3):196-202.

[13] Giacomozzi C, Caselli A, Macellari V, Giurato L, Lardieri L, Uccioli L. Walking strategy in diabetic patients with peripheral neuropathy. Diabetes Care. 2002; 25(8):1451-457.

[14] Shaw JE, Van Schie C, Carrington A, Abbott C, Boulton A. An analysis of dynamic forces transmitted through the foot in diabetic neuropathy. Diabetes Care. 1998; 21(11):1955-959.

[15] Katoulis EC, Ebdon-Parry M, Lanshammar H, Vileikyte L, Kulkarni J, Boulton AJM. Gait abnormalities in diabetic neuropathy. Diabetes Care. 1997; 20(12):1904-907.

[16] Mueller MJ, Minor SD, Sahrmann SA, Schaaf JA, Strube MJ. Differences in the gait characteristics of patients with diabetes and peripheral neuropathy compared with age-matched controls. Physical Therapy. 1994; 74(4):299-308. $\left.[1], 1 T_{6}\right) \Delta_{6}$

زمان مرحله ايستايي بهعنوان شاخصى مهم در بررسى تأثير

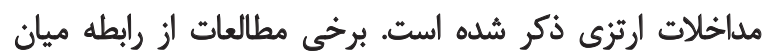

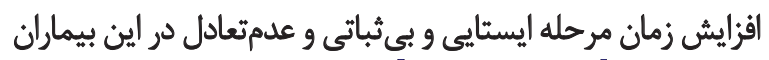

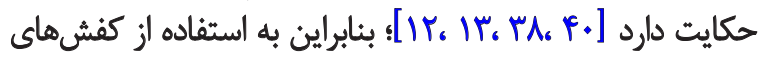

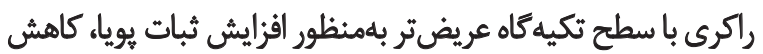

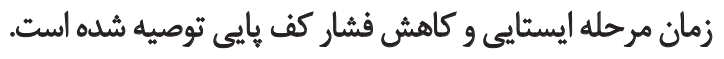

همجينين، اين مداخلات ممكن است نواحى اعمال نيرو را افزايش

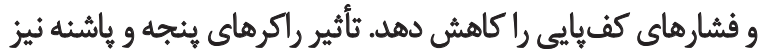

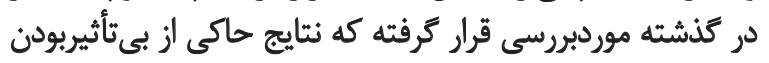

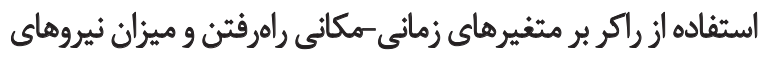

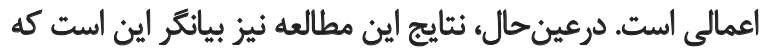

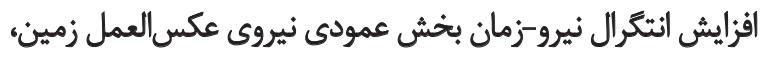

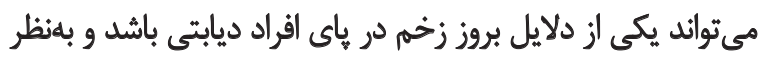

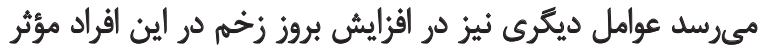

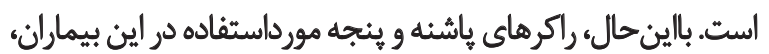
بر ميزان نيروهاى اعمالى و انتكرال نيرو-زمان بان تأثيرى نداشته است.

$$
\text { تئيجليرى }
$$

راكرهاى ياشنه و ينجه مورداستفاده در اين بيماران، بر ميزان

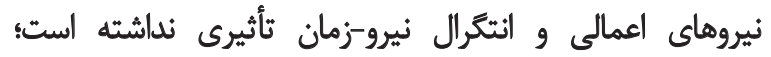

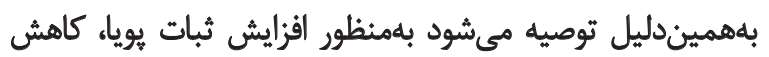

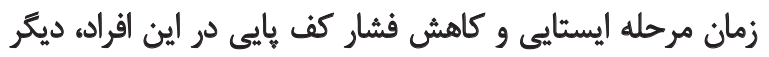

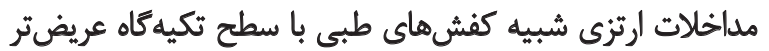
مورداستفاده قرار كيرد.

محدوديتها و يشئهادها

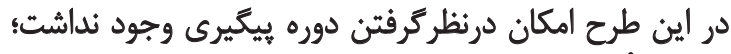

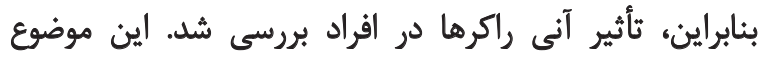

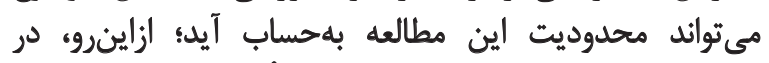

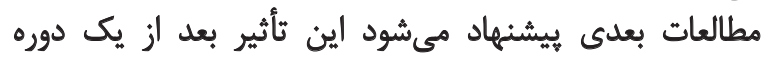
استفاده نيز موردارزيابي قرار كيرد.

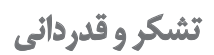

اين مقاله حامى مالى نداشته است. 
[17] Bus SA, Van Deursen R, Kanade RV, Wissink M, Manning EA, van Baal JG, et al. Plantar pressure relief in the diabetic foot using forefoot offloading shoes. Gait \& Posture. 2009; 29(4):618-22.

[18] Myers K, Long J, Klein J, Wertsch J, Janisse D, Harris G. Biomechanical implications of the negative heel rocker sole shoe: Gait kinematics and kinetics. Gait \& Posture. 2006; 24(3):323-30.

[19] Owings TM, Woerner JL, Frampton JD, Cavanagh PR, Botek G. Custom therapeutic insoles based on both foot shape and plantar pressure measurement provide enhanced pressure relief. Diabetes Care. 2008; 31(5):839-44.

[20] Reiber GE, Smith DG, Boone DA, Del Aguila M, Borchers RE, Mathews D, et al. Design and pilot testing of the DVA/Seattle Footwear System for diabetic patients with foot insensitivity. Journal of rehabilitation research and development. 1997; 34(1):1-8.

[21] Bus SA, Ulbrecht JS, Cavanagh PR. Pressure relief and load redistribution by custom-made insoles in diabetic patients with neuropathy and foot deformity. Clinical Biomechanics. 2004; 19(6):629-38.

[22] Lavery LA, Vela SA, Fleischli JG, Armstrong DG, Lavery DC. Reducing plantar pressure in the neuropathic foot: a comparison of footwear. Diabetes Care. 1997; 20(11):1706-710.

[23] Guldemond N, Leffers P, Schaper N, Sanders A, Nieman F, Willems $\mathrm{P}$, et al. The effects of insole configurations on forefoot plantar pressure and walking convenience in diabetic patients with neuropathic feet. Clinical Biomechanics. 2007; 22(1):81-87.

[24] Bus SA, Haspels R, Busch-Westbroek TE. Evaluation and optimization of therapeutic footwear for neuropathic diabetic foot patients using in-shoe plantar pressure analysis. Diabetes Care. 2011; 34(7):1595-1600.

[25] Praet SFE, Louwerens JWK. The influence of shoe design on plantar pressures in neuropathic feet. Diabetes Care. 2003; 26(2):441-45.

[26] Kavros SJ, Van Straaten MG, Coleman Wood KA, Kaufman KR. Forefoot plantar pressure reduction of off-the-shelf rocker bottom provisional footwear. Clinical Biomechanics. 2011; 26(7):778-82.

[27] Giacalone VF, Armstrong DG, Ashry HR, Lavery DC, Harkless LB, Lavery LA. A quantitative assessment of healing sandals and postoperative shoes in offloading the neuropathic diabetic foot. Journal of Foot and Ankle Surgery. 1997; 36(1):28-30.

[28] Long JT, Klein JP, Sirota NM, Wertsch JJ, Janisse D, Harris GF. Biomechanics of the double rocker sole shoe: gait kinematics and kinetics. Journal of Biomechanics. 2007; 40(13):2882-890.

[29] Perry SD, Radtke A, Mcllroy WE, Fernie GR, Maki BE. Efficacy and effectiveness of a balance-enhancing insole. Journals of Gerontology Series A: Biological Sciences and Medical Sciences. 2008; 63(6):595-602.

[30] Kirtley C. Clinical gait analysis: theory and practice: New York: Elsevier Health Sciences; 2006.

[31] Mueller MJ, Diamond JE. Biomechanical treatment approach to diabetic plantar ulcers. Physical Therapy. 1988; 68(12):1917-920.
[32] Bahramian H, Ghoseiri K. [Assessment of the foot plantar pressure in type II diabetic patients with mild neuropathy (Persian)]. Journal of Rehabilitation. 2011; 12(2):34-40.

[33] Safaei-Pour Z, Ebrahimi E, Saeedi H, Kamali M. [Investigation of dynamic plantar pressure distribution in healthy adults during standing and walking (Persian)]. Journal of Rehabilitation. 2009; 10(2):8-15.

[34] Farjad-Pezeshk A, Sadeghi H, Farzadi M. [Comparison of plantar pressure distribution and vertical ground reaction force between dominant and none-dominant limb in healthy subjects using principle component analysis (PCA) technique (Persian)]. Journal of Rehabilitation. 2013; 14(1):91-102.

[35] Van Schie C, Ulbrecht J, Becker M, Cavanagh P. Design criteria for rigid rocker shoes. Foot and Ankle International. 2000; 21(10):833-44.

[36] Chapman J, Preece S, Braunstein B, Hohne A, Nester C, Brueggemann $\mathrm{P}$, et al. Effect of rocker shoe design features on forefoot plantar pressures in people with and without diabetes. Clinical Biomechanics. 2013; 28(6):679-85.

[37] Esrafilian A, Karimi MT, Eshraghi A. Design and evaluation of a new type of knee orthosis to align the mediolateral angle of the knee joint with osteoarthritis. Advances in Orthopedics. 2012; 1:6. doi: $10.1155 / 2012 / 104927$

[38] Wrobel JS, Najafi B. Foot Technology, Part 1 of 2: diabetic foot Biomechanics and gait dysfunction. Journal of Diabetes Science and Technology. 2010; 4(4):833-45.

[39] Sawacha Z, Gabriella G, Cristoferi G, Guiotto A, Avogaro A, Cobelli C. Diabetic gait and posture abnormalities: A biomechanical investigation through three dimensional gait analysis. Clinical Biomechanics. 2009; 24(9):722-28.

[40] Allet L, Armand S, Golay A, Monnin D, De Bie R, De Bruin E. Gait characteristics of diabetic patients: a systematic review. Diabetes \& Metabolism Research and Reviews. 2008; 24(3):173-91.

[41] Mueller MJ. Therapeutic footwear helps protect the diabetic foot. Journal of the American Podiatric Medical Association. 1997; 87(8):360-4.

[42] Rao S, Saltzman C, Yack HJ. Ankle ROM and stiffness measured at rest and during gait in individuals with and without diabetic sensory neuropathy. Gait \& Posture. 2006; 24(3):295-301.

[43] Hatef B, Bahrpeyma F, Mohajeri Tehrani MR. [The relationship between duration of type two diabetes and knee muscles strength (Persian)]. Specific Physical Therapy Journal. 2013; 3(2):34-40. 
